\title{
Savings after extinction in conditioning of the rabbit's nictitating membrane response
}

\author{
MICHAELA MACRAE and E. JAMES KEHOE \\ University of New South Wales, Sydney, New South Wales, Australia
}

\begin{abstract}
The present experiments examined two forms of savings after the extinction of a conditioned response (CR) in the rabbit nictitating membrane preparation. Each experiment entailed three groups. First, a reacquisition (RAQ) group received initial conditioned stimulus-unconditioned stimulus (CS-US) acquisition, followed by CS-alone extinction, and then CS-US reacquisition with the same stimulus. Second, a cross-modal acquisition (CMA) group received initial acquisition and extinction with one stimulus but received its final stage of training with a stimulus from another modality. Third, a naive acquisition group (designated as REST) received only the final stage of CS-US training. In all three groups, tone and light were used as CSs in a counterbalanced fashion. In addition, the experiments tested whether the manipulation of contextual factors-namely, trial spacing and the number of trials per session-could influence the rates of acquisition in Groups RAQ and CMA after extinction. The experiments demonstrated that (1) reacquisition of the CR to the original CS was very rapid relative to Group REST, (2) cross-modal acquisition was also rapid relative to Group REST, and (3) in Group CMA, tests of the original CS showed concurrent recovery, which is a new phenomenon and is distinct from spontaneous recovery. However, the relative rates of acquisition after extinction appeared constant across the contextual manipulations. The results are discussed with respect to the relative stability of excitatory and inhibitory learning, as well as two alternative theories of savings after extinction.
\end{abstract}

The present experiments were aimed at assessing savings after the extinction of a conditioned response (CR). Savings have been demonstrated most prominently in the rabbit nictitating membrane (NM) preparation in studies of reacquisition after extinction and cross-modal acquisition after extinction. In reacquisition, when the original conditioned stimulus (CS) is again paired with the unconditioned stimulus (US), recovery of the CR is more rapid than acquisition in a rest control group (Napier, Macrae, \& Kehoe, 1992). In cross-modal acquisition, a new CS from a different modality is introduced after extinction. Subjects' acquisition to the new CS is significantly more rapid than that of naive controls (Kehoe, Horne, \& Macrae, 1995; Kehoe, Morrow, \& Holt, 1984).

A review of reacquisition in other animal learning preparations, however, reveals that the relative rates of reacquisition, cross-modal acquisition, and naive acquisition are highly variable. Some of the studies that compared reacquisition with a rest control have found that reacquisition can be faster than naive acquisition. This was the case in three different learning preparations that used ratsnamely, an escape from fear task (McAllister \& McAllister, 1994), appetitive conditioning (Ricker \& Bouton, 1996, Experiments 1a, 2, and 3), and taste aversion learning (Revusky \& Coombes, 1979). On the other hand, reacqui-

This research was supported by Australian Research Council Grant A79600502 to E.J.K. The authors thank Anastasia Georgilas and Gabrielle Weidemann for their assistance in the collection and analysis of the data. Correspondence should be addressed to M. Macrae, School of Psychology, University of New South Wales, Sydney, NSW 2052, Australia (e-mail: m.macrae@unsw.edu.au). sition has also been found to be slower than naive acquisition in conditioned suppression in rats (Bouton, 1986).

Comparisons of reacquisition and cross-modal acquisition have found reacquisition to be slower than crossmodal acquisition both in conditioned suppression in rats (Bouton, 1986) and in taste aversion learning (Hart, Bourne, \& Schachtman, 1995). However, in appetitive learning in rats, rates of reacquisition and cross-modal acquisition were equivalent (Ricker \& Bouton, 1996). There are only two direct comparisons of cross-modal acquisition with naive acquisition. Cross-modal acquisition has been demonstrated as faster than naive acquisition in the rabbit NM preparation (Kehoe et al., 1995) and in appetitive learning in rats (Ricker \& Bouton, 1996). This latter type of comparison has been infrequent because the traditional experimental focus has been on reacquisition; cross-modal acquisition has tended to function as a learning-experienced control condition that equates prior exposure to CS-US pairings and extinction. Evidence that cross-modal acquisition can yield savings in its own right (e.g., Kehoe et al., 1995) points to the need to include naive or "rest" control conditions.

To date, variability in the rate of reacquisition has been attributed to preparation-specific differences that were, by implication, unresolvable (e.g., Bouton, 1986; Napier et al., 1992). However, a recent paper by Ricker and Bouton (1996) has indicated that the rate of reacquisition depends not simply on the animal preparation used but, rather, on the number and spacing of trials in the acquisition and extinction phases. Ricker and Bouton conducted a three-way comparison among reacquisition, cross-modal acquisition, and naive acquisition, using ap- 
petitive conditioning in rats. In Experiment 1, both reacquisition and cross-modal acquisition were significantly faster than acquisition in a naive control group. In Experiments 2 and 3 , the reacquisition phase consisted of a partial reinforcement schedule, rather than $100 \%$ reinforced trials, but, again, both reacquisition and cross-modal acquisition were facilitated relative to naive controls. In Experiment 4, the conditioning and reacquisition phases contained fewer trials that were delivered in a widely spaced fashion. This manipulation reduced the rate of reacquisition such that it was slower than in the rest control condition. Cross-modal acquisition was also reduced and appeared equivalent to naive acquisition in a rest control.

As a general hypothesis, the experimental context of learning after extinction may determine the rate of that learning (Bouton, 1993). More specifically, the context in which learning after extinction occurs might retrieve both excitatory associations formed during acquisition and inhibitory associations formed during extinction. Consequently, the rates of reacquisition and cross-modal acquisition would be determined in part by the relative number of initial acquisition and extinction trials. The rates would also be determined by the spacing of those trials, if trial spacing acted as a contextual cue. For example, if reacquisition trials were delivered at the same pace as initial acquisition trials, retrieval of an excitatory association would be facilitated. Conversely, if reacquisition trials were delivered at the same pace as extinction trials, retrieval of inhibitory associations would be facilitated.

These recent findings point to the need for a three-way comparison among reacquisition, cross-modal acquisition, and naive acquisition in the rabbit NM preparation. Although reacquisition and cross-modal acquisition have shown savings when compared with a naive control group, those two experimental conditions have never been directly compared in the rabbit. This kind of comparison would, first of all, help to reconcile the massive savings seen in the rabbit NM preparation versus the interference with reacquisition seen in some other preparations. Thus, in the present study, Experiment 1 was a three-way comparison of reacquisition, cross-modal acquisition, and naive acquisition in the rabbit NM preparation, in which all stages of training contained the same number and spacing of trials. Experiment 2 was also a comparison of reacquisition, cross-modal acquisition, and naive acquisition, but, as will be explained, the number and spacing of trials varied across the three stages.

A second aim of the present experiments was to examine whether the rates of reacquisition and cross-modal acquisition in the NM preparation could be influenced by manipulating the number and spacing of trials per session in the acquisition and extinction phases of training (cf. Ricker \& Bouton, 1996). More generally, the experiments sought to test the hypothesis that the retrieval of excitatory and inhibitory associations during reacquisition can be influenced by altering the context of reac- quisition to make it either more or less similar to the contexts of initial acquisition and extinction. Because previous experiments examining reacquisition in the rabbit NM preparation have indicated that excitatory strength tends to be predominant (e.g., Napier et al., 1992), the present manipulations were aimed at increasing the likelihood that inhibition would prevail in Stage 3. To this end, Experiment 2 used spaced trials in Stage 1 and massed trials in Stages 2 and 3.

A third aim was to determine the fate of the original CS in cross-modal acquisition. In the rabbit NM preparation, there has been some previous evidence that when the original CS is tested during cross-modal training, "concurrent recovery" occurs. That is, CRs to the original CS recover, despite the fact that the original CS is not paired with the US (Kehoe et al., 1995). However, this kind of recovery has not been reported elsewhere. Therefore, the final stages of Experiments 1 and 2 contained test trials of the original CS among training trials with a novel CS.

\section{EXPERIMENT 1}

Experiment 1 was a three-way comparison of reacquisition, cross-modal acquisition, and naive acquisition in the rabbit NM preparation. The reacquisition (RAQ) group received initial CS-US acquisition followed by CS-alone extinction and then CS-US reacquisition with the same stimulus. The cross-modal acquisition (CMA) group received initial acquisition and extinction with one stimulus but received its final stage of training with a stimulus from another modality. The naive acquisition group was designated as REST. This group received exposure to the apparatus during the first two stages of training and received CS-US pairings in the final stage of training (Marchant \& Moore, 1974; Napier et al., 1992). In all three groups, tone and light were used as the stimuli in a counterbalanced fashion.

Each session in Stage 3 contained trials that tested for generalization between the tone and light stimuli and for concurrent recovery. Thus, the animals that were given reacquisition training with their original CS (say, tone) were also tested with a stimulus that was novel and never reinforced (i.e., light). The same was true for the animals in the rest control condition. That is, the test stimulus was novel and never reinforced. In cross-modal training, however, the test stimulus was, in fact, the original CS. Portions of the results have been reported previously in abstract form (Kehoe \& Macrae, 1997).

\section{Method}

Subjects. The subjects were 24 , naive female albino rabbits (Oryctolagus cuniculus), weighing approximately $1.5 \mathrm{~kg}$ on arrival from the university's breeding unit. All rabbits had free access to food and water in their home cages.

Apparatus. The apparatus and recording procedure for the NM response were patterned after those of Gormezano (1966) and are detailed by Kehoe, Feyer, and Moses (1981). The rabbits were trained in one of eight sound-attenuating chambers. On the wall of 

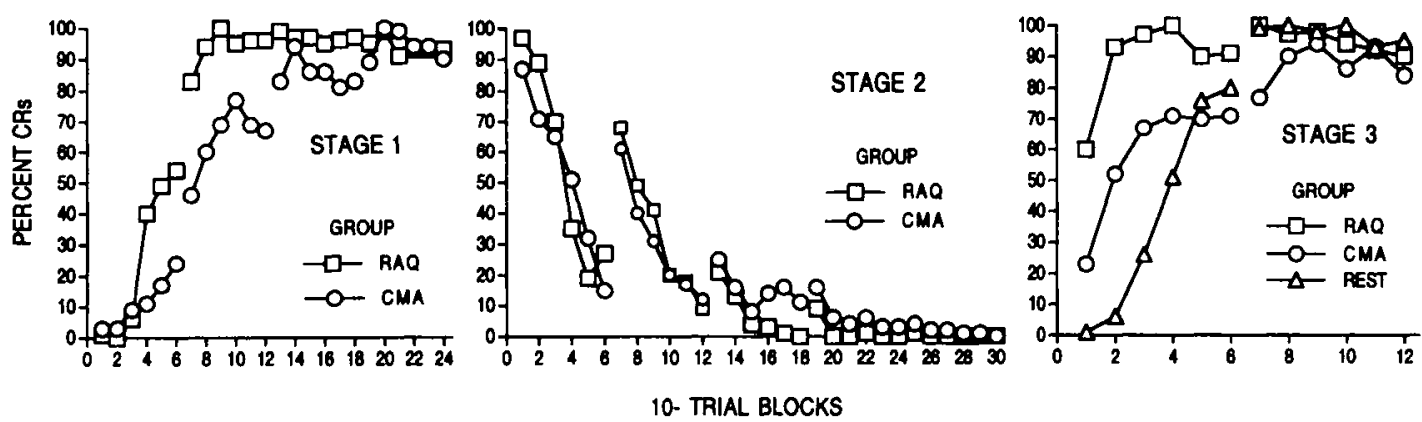

Figure 1. Percentage CRs of Groups RAQ, CMA, and REST as a function of 10-trial blocks in Stages 1, 2, and 3 of Experiment 1.

the chamber in front of each subject was a stimulus panel, and behind the subject was an exhaust fan. A speaker was mounted at the midpoint of the stimulus panel, $8 \mathrm{~cm}$ anterior to and $16 \mathrm{~cm}$ above the rabbit's head. The speaker provided an auditory CS that was a $1000-\mathrm{Hz}, 88-\mathrm{dB}$ tone of $250-\mathrm{msec}$ duration superimposed on an ambient noise level of $81 \mathrm{~dB}$. Mounted on the stimulus panel, $4 \mathrm{~cm}$ above the speaker was an 8 -W neon light that served as a houselight. A visual CS was provided by a $20-\mathrm{Hz}$ flashing of the houselight for $250 \mathrm{msec}$. The US was a $50-\mathrm{msec}, 3-\mathrm{mA}, 50-\mathrm{Hz}$ ac electric current delivered via 9-mm stainless steel Autoclip wound clips positioned $10 \mathrm{~mm}$ apart and $15 \mathrm{~mm}$ posterior to the dorsal canthus of the rabbit's right eye. The sequence and timing of stimulus events were controlled by an Apple II computer equipped with interfaces and software developed by Scandrett and Gormezano (1980).

During training, each rabbit was restrained in a Perspex box $(45$ $\times 14 \times 22 \mathrm{~cm}$ ). The rabbit was held in place by insertion of its head through an adjustable stock and by securing its ears to the front of the stock with a polyurethane foam-covered metal clamp. A muzzlelike head set, fitted securely about the snout, supported a photoelectric transducer for detecting movements of the NM (Gormezano $\&$ Gibbs, 1988). A small tinned copper wire hook was attached to a silk loop sutured in the NM of the rabbit's right eye. The other end of the hook contained a loop that fitted over the curved end of an Lshaped piano-wire crank, which operated the transducer. The signal from the transducer was amplified and transmitted to an analog/digital converter installed in the computer.

Procedure. All rabbits received 1 day of preparation, 2 days of recovery, and 1 day of adaptation before training began. On the day of preparation, hair posterior to the rabbit's right eye was removed, a small loop of silk (000 Dynex) was sutured into the NM of the right eye, and the rabbits were returned to their home cages for 2 days of recovery. On the adaptation day, the rabbits were placed in the conditioning apparatus for $60 \mathrm{~min}$, but no stimuli were presented.

On the day following adaptation, the rabbits were randomly assigned to one of three groups $(n=8)$ designated RAQ, CMA, and REST according to their treatment in the final stage of training. In brief, Group RAQ received reacquisition training with its original CS, Group CMA received cross-modal acquisition with a new CS, and Group REST was the naive, rest control group.

In Stage 1, which lasted for 3 days, Groups RAQ and CMA received $60 \mathrm{CS}-\mathrm{US}$ trials per day. For half the animals in each group, the CS was the tone. For other half, the CS was the light. The CSUS interval (onset to onset) was $250 \mathrm{msec}$. The mean intertrial interval (ITI) was $60 \mathrm{sec}$ (range $50-70 \mathrm{sec}$ ). Group REST was placed in the conditioning apparatus for $60 \mathrm{~min}$ but received neither the CS nor the US.

In Stage 2, which lasted for 5 days, both Groups RAQ and CMA received $60 \mathrm{CS}$-alone extinction trials per session at a mean ITI of
$60 \mathrm{sec}$. Group REST continued to sit restrained in the conditioning apparatus.

In Stage 3, which lasted for 2 days, all groups received $60 \mathrm{CS}-$ US trials per session. In Group RAQ, each subject received training with its original CS. In Group CMA, each subject received training with the alternative CS. Thus, the animals trained originally with the tone $C S$ received training with the light $C S$, or vice versa. In Group REST, half the animals received training with the tone CS, and half the animals received training with the light CS. For all groups, the CS-US interval was $250 \mathrm{msec}$, and the mean ITI was $60 \mathrm{sec}$. In addition to the CS-US trials, each session contained 3 nonreinforced trials for the alternative stimulus. A test trial was presented after the 10th, 30th, and 50th CS-US trial.

Response definition. A CR was defined as any extension of the $\mathrm{NM}$ greater than or equal to $0.5 \mathrm{~mm}$ that occurred following the onset of the CS but prior to its termination (Marshall-Goodell, Schreurs, \& Gormezano, 1982).

\section{Results}

Unless otherwise noted, planned contrasts were used to analyze the data, and the rejection level was set according to a Type I error rate of .05. Because Experiment 1 focused on responding in Stage 3, rigorous criteria were applied to ensure that each subject had shown both CR acquisition by the end of Stage 1 and complete CR extinction by the end of Stage 2. Two animals failed to meet these criteria: 1 animal in Group RAQ did not show any initial CR acquisition, and 1 animal in Group CMA persisted in responding throughout all 5 days of extinction training. Accordingly, their data were excluded from the analysis and presentation of the results.

Figure 1's left panel shows the mean percentage of CRs for Groups RAQ and CMA plotted across the 3 days of Stage 1. Each day is divided into six blocks of 10 trials. The points between each set of six blocks are not connected in order to represent the 24-h gap between them. The curves show that both groups acquired CRs to asymptotic levels exceeding 90\% CRs. Although Group RAQ appeared to show faster CR acquisition than Group CMA, this difference was not statistically significant in either overall comparisons between the groups or interactions with trial blocks [largest $F(1,12)=3.13, p>.10$ ].

Figure 1's center panel shows the mean percentage of CRs in 10-trial blocks across the 5 days of Stage 2. Groups 


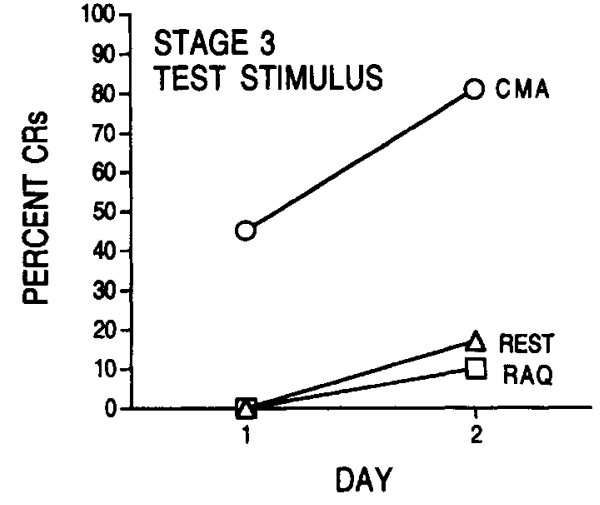

Figure 2. Percentage CRs on tests of the alternative stimulus in Groups RAQ, CMA, and REST as a function of days in Stage 3 of Experiment 1.

RAQ and CMA showed extinction interspersed with spontaneous recovery early in Stage 2 . By the last day of Stage 2, extinction appeared complete. The highest mean level of responding in any trial block was $4 \% \mathrm{CRs}$, which is consistent with the base level of spontaneous responding in the NM preparation (Gormezano, Kehoe, \& Marshall, 1983). Any apparent differences between the two groups were small, unsystematic, and not statistically significant $(F \mathrm{~s}<1)$.

Figure 1's right panel shows CR acquisition in Stage 3 for all three groups. The means are plotted in 10-trial blocks, but the scale has been expanded in order to better see the acquisition curves. Rapid reacquisition in Group RAQ was clearly evident on the 1st day of Stage 3. For example, in the first block of trials, Group RAQ showed a mean level of $60 \%$ CRs, which was much higher than that of either Group CMA ( $M=23 \%$ CRs) or Group REST $(M=1 \%$ CRs). In addition, Group CMA showed a facilitation in its rate of $\mathrm{CR}$ acquisition indicative of cross-modal savings. Throughout most of the 1 st day, Group CMA's level of responding was higher than that of Group REST. On the 2nd day, responding in all three groups converged to mean levels around $90 \% \mathrm{CRs}$.

Statistical comparisons confirmed the apparent differences among the groups. Over all of Stage 3, Group RAQ's responding ( $M=92 \%$ CRs) was significantly greater than either Group CMA $(M=73 \%$ CRs $)[F(1,19)=$ $4.69, p<.05]$ or Group REST $(M=69 \%$ CRs $)[F(1,19)=$ $7.72, p<.05]$. More importantly, the slope of Group RAQ's learning curve differed significantly from those of the other two groups [linear-trend $F \mathrm{~s}(1,19)=6.31$ and $34.07, p s<.05]$. Comparisons between Group CMA and Group REST revealed a significant difference in the slopes of their learning curves $[F(1,19)=10.51, p<.05]$ but not their overall means $(F<1)$.

The direction of the difference between Groups RAQ and CMA in Stage 3 paralleled the difference that was apparent, but not significant, in Stage 1. That is, in both stages, Group RAQ showed greater responding than
Group CMA. Inspection of the data from Stage 1 revealed that two rabbits in Group CMA showed very low levels of initial acquisition. Over the 4 days of Stage 1, these 2 rabbits showed levels of $30 \%$ and $46 \% \mathrm{CRs}$, whereas the lowest level shown by any rabbit in Group RAQ was $63 \%$ CRs. When the performance of these 2 rabbits was removed from the Stage 3 data, the overall performance of the remaining 5 rabbits in Group CMA more closely approximated the high level of responding seen in Group RAQ. That is, on the 1st day of Stage 3, Group CMA's level of responding ( $M=69 \% \mathrm{CRs}$ ) failed to differ significantly from that of Group RAQ $(M=88 \% \mathrm{CRs})[F(1,10)=$ $2.67, p>.10]$.

Figure 2 shows the results of the tests with the alternative stimulus. In Group CMA, the CR to the original CS reappeared on test trials. On the 1st day of Stage 3,6 of the 7 animals in Group CMA showed at least one CR to the original CS, and, on the 2nd day, all the animals in Group CMA responded to the test stimulus. In contrast, Groups RAQ and REST showed little responding to what for them was a novel stimulus. On the 1st day of Stage 3, no animal in either group responded to the test stimulus. On the 2nd day, only 1 animal in Group RAQ responded and 2 in Group REST responded.

Statistical comparisons confirmed that Group CMA's level of responding to the test stimulus ( $M=63 \% \mathrm{CRs}$ ) was significantly greater than either Group RAQ $(M=5 \%$ CRs) or Group REST $(M=8 \% \mathrm{CRs})[F \mathrm{~s}(1,19)=37.81$ and $35.53, p \mathrm{~s}<.05]$. There was also a significant rise in the level of responding between the 1st and 2nd day of Stage $3[F(1,19)=13.63, p<.05]$. This rise did not interact with any of the group comparisons - that is, the group $\times$ session interaction was not significant. Finally, any apparent differences between Groups RAQ and REST were not significant.

\section{Discussion}

Experiment 1 resulted in two major findings. First, reacquisition and cross-modal acquisition were both faster than acquisition in a rest control. The difference between reacquisition and cross-modal acquisition was ambiguous, but reacquisition was at least as fast as, if not faster than, cross-modal acquisition. Second, in conjunction with cross-modal acquisition, Group CMA showed recovery of responding to the original CS.

The rapid reacquisition seen in this experiment confirmed previous demonstrations by Napier et al. (1992). As in Napier et al., care was taken to eradicate spontaneous recovery as a possible source of rapid reacquisition by the use of prolonged extinction training. Complete extinction was achieved in 5 days, after which CS-US pairings were resumed and yielded the rapid reappearance of the $\mathrm{CR}$. The demonstration that cross-modal acquisition was faster than acquisition in a rest control was also consistent with previous findings in the NM preparation (Kehoe et al., 1995; Kehoe et al., 1984).

A new finding was that reacquisition was at least equivalent to, if not faster than, cross-modal acquisition. 
These two forms of savings have not been directly compared in the rabbit NM preparation previously. In other preparations, reacquisition has variously been found to be slower than cross-modal acquisition (Bouton, 1986; Hart et al., 1995) or the same as cross-modal acquisition (Ricker \& Bouton, 1996). A hypothesis that goes part of the way toward explaining the present findings is that, for Group RAQ, Stage 3 involved a return to the original context of acquisition, therefore causing the animals to retrieve a memory of responding to that particular CS. For Group CMA, the pairing of a new CS with the US in Stage 3 might have (1) caused a release from inhibition because of the presence of the new CS and (2) caused retrieval of a memory of responding because of the return of parts of the context of acquisition (e.g., the US). However, if this hypothesis were entirely correct, it would be expected that cross-modal acquisition would be faster than reacquisition. The present findings suggest that there is some other unknown factor involved that either slows cross-modal acquisition or enhances the rate of reacquisition.

Group CMA also demonstrated concurrent recovery. For example, the animals that were originally trained with a tone, were extinguished with the same tone, and were then transferred to light-US pairings showed a progressive increase in responding on unreinforced tone tests as they acquired CRs to the light. This recovery of the original CR in Group CMA was not simply generalization between the tone and the light, because there was negligible evidence of CRs to the test stimulus in either Group RAQ or Group REST in Stage 3. Had there been weak generalization across modalities, it would have appeared in Group RAQ because, first, it received the same pattern of initial CS-US training and CS-alone extinction as Group CMA, and, second, Group RAQ showed a higher level of responding than Group CMA in Stage 3, thus providing a good potential for generalized responses to the test stimulus.

\section{EXPERIMENT 2}

Experiment $2 \mathrm{~A}$ was aimed at reducing retrieval of excitatory associations and maximizing the likelihood of inhibitory associations being expressed during Stage 3 training. The context of Stage 1 acquisition training was made distinct from the context of Stage 3 training by reducing the total number of trials and lengthening their spacing. Conversely, the context of Stage 2 extinction training was kept as similar as possible to the context of Stage 3 training by presenting the same number and spacing of trials in both stages.

Experiment 2B was a control experiment that was the reverse of Experiment 2A. Massed trials were used in Stages 1 and 2, whereas spaced trials were used in Stage 3. At an empirical level, the results of this reversal could be compared with those of Experiment $2 \mathrm{~A}$ in order to reveal any asymmetry in transfer between spaced and massed CS-US training. At a more theoretical level, the use of spaced training made Stage 3 distinct from Stages 1 and 2. The retrieval of memories from both earlier stages might be reduced, thus possibly eliminating the advantage of reacquisition and cross-modal acquisition relative to naive acquisition. However, it is widely accepted that inhibition is more labile than excitation (Pavlov, 1927, p. 66), and, by the same token, inhibition might be particularly sensitive to changes in context. In that case, the change in trial spacing from Stage 2 to Stage 3 might especially reduce the expression of inhibition and thus allow the excitatory associations established in initial acquisition to predominate.

Trial spacing was manipulated by simultaneously altering the number of trials per session and the ITI within sessions. In our spaced training, there were 5 CS-US trials per session presented at an average ITI of $10 \mathrm{~min}$. In massed training, there were $60 \mathrm{CS}-\mathrm{US}$ trials per session separated by an average ITI of $60 \mathrm{sec}$.

\section{Method}

Subjects. The subjects were 48 naive male and female albino rabbits.

Apparatus and Procedure. Unless otherwise indicated, the apparatus and procedures were identical to those of Experiment 1. In Experiments $2 \mathrm{~A}$ and $2 \mathrm{~B}$, the rabbits were randomly assigned to three groups $(n=8)$ designated as RAQ, CMA, and REST.

In Experiment 2A, Groups RAQ and CMA received 5 CS-US trials per session in Stage 1. The mean ITI was $10 \mathrm{~min}( \pm 1 \mathrm{~min})$. The animals were trained for at least 7 sessions or until they reached a criterion of $75 \%$ CRs. No animal required more than 9 sessions to reach this criterion. In Stage 2, Groups RAQ and CMA each received 5 sessions of massed CS-alone extinction (60 CS presentations per session separated by a mean ITI of $60 \mathrm{sec}$ ). In Stages 1 and 2 , the animals in Group REST remained in their home cages (Marchant \& Moore, 1974). Finally, in Stage 3, Groups RAQ, CMA and REST received massed CS-US training ( 60 trials per session separated by a mean ITI of $60 \mathrm{sec}$ ). Group RAQ received training with its original $\mathrm{CS}$, and Group CMA received training with a new CS. All groups also received three nonreinforced test presentations of the alternative stimulus in each session of Stage 3. As in Experiment 1 , the roles of the tone and light CSs were counterbalanced within each group. In Group REST, half the animals received training with the tone $C S$, and half received training with the light $C S$.

In Experiment 2B, Groups RAQ and CMA received 3 days of massed CS-US training in Stage 1 and 5 sessions of massed CSalone extinction in Stage 2. In Stages 1 and 2, Group REST was restrained in the experimental chambers. In Stage 3, all three groups received spaced CS-US training. Group RAQ received training with its original CS, and Group CMA received training with a new CS. As a consequence of the small number of trials per session, Stage 3 did not contain any tests with the alternative stimulus. The roles of the tone and light CSs were counterbalanced within each group.

\section{Results}

Experiment 2A (spaced-massed-massed). The data from 3 animals were excluded from the analyses using the same criteria for acquisition and extinction used in Experiment 1. One animal in Group RAQ failed to show any CRs in Stage 1, and 2 animals failed to show complete extinction in Stage 2 ( 1 each in Group RAQ and Group CMA).

Figure 3 depicts $\mathrm{CR}$ performance during the three stages of Experiment $2 \mathrm{~A}$. In the left panel, the data for 

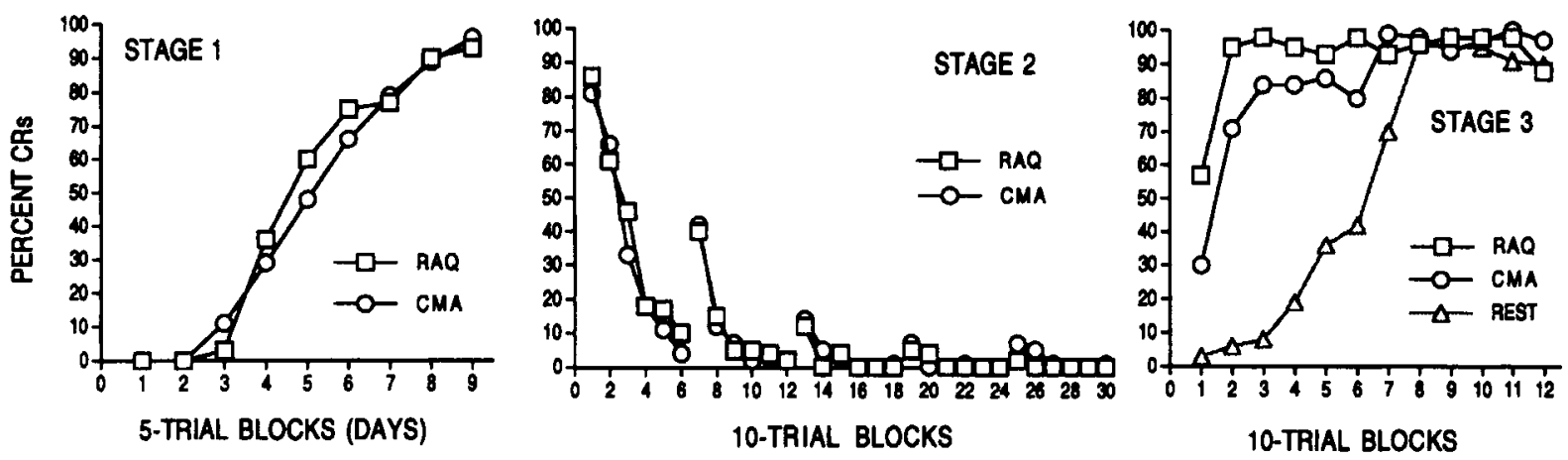

Figure 3. Percentage CRs of Groups RAQ, CMA, and REST in Experiment 2A. For Stage 1, the data are plotted in 5-trial blocks, 1 for each day of training. For Stages 2 and 3, the data are plotted in 10-trial blocks, 6 for each day of training.

Stage 1 are plotted in 5-trial blocks. For these animals that received less than 9 days of training, their level of responding was extrapolated up to Day 9 . As can be seen, Groups RAQ and CMA achieved mean levels around $95 \%$ CRs within 45 trials. Any apparent differences were small and not significant $(F \mathrm{~s}<1)$.

The center panel of Figure 3 shows the course of CR extinction plotted in blocks of 10 trials. By the last day of Stage 2, extinction was complete in both groups; the highest mean level of responding in any trial block was only $7 \%$ CRs. Any apparent differences between the two groups were not statistically significant $\left(F_{\mathrm{S}}<1\right)$.

Figure 3's right panel shows CR acquisition in Stage 3 plotted in 10-trial blocks. The pattern of results closely resembled those of Experiment 1. That is, Group RAQ's rate of reacquisition appeared faster than that of crossmodal acquisition in Group CMA. In turn, both of these groups showed a faster rise in responding than Group REST.

Statistical comparisons between Group RAQ and Group CMA revealed a significant difference in the slopes of their learning curves $[F(1,18)=4.40, p<.05]$, but not their overall means $(F<1)$. The overall means of both Group RAQ $(M=92 \% \mathrm{CRs})$ and Group CMA $(M=85 \%$ CRs) were significantly greater than that of Group REST $(M=54 \%$ CRs $)[F \mathrm{~s}(1,18)=28.35$ and $20.16, p s<.05]$. Moreover, the slopes of Groups RAQ's and CMA's learning curves differed significantly from that of Group REST [linear-trend $F \mathrm{~s}(1,18)=46.55$ and $23.70, p \mathrm{~s}<.05$ ].

Figure 4 shows the results of the tests with the alternative stimulus during Stage 3 in Experiment $2 \mathrm{~A}$. As seen in Experiment 1, Group CMA recovered responding to its original CS concurrent with CR acquisition to the new CS. On both days of Stage 3, 5 of the 7 animals in Group CMA showed at least one CR to the original CS. In contrast, Groups RAQ and REST showed little responding to the test stimulus. No animal in either group responded on the 1 st day of Stage 3. On the 2nd day, only 1 animal in each of those groups responded to the test stimulus.

Statistical comparisons confirmed that Group CMA's level of responding to the test stimulus ( $M=54 \%$ CRs) was significantly greater than that in either Group RAQ $(M=8 \% \mathrm{CRs})$ or Group REST $(M=2 \% \mathrm{CRs})[F \mathrm{~s}(1,18)=$ 9.66 and 14.47 , ps <.05]. Any apparent difference between Groups RAQ and REST was not significant. Unlike in Experiment 1, there was no significant rise in the level of responding between the 1st day and the 2 nd day of Stage 3. Responding in each group reached its maximum level within the 1st day of Stage 3.

Experiment 2B (massed-massed-spaced). The data from 2 animals were excluded from the analyses. One animal in Group CMA failed to acquire CRs in Stage 1, and 1 animal in Group RAQ failed to extinguish CRs in Stage 2.

Figure 5 depicts $C R$ performance during the three stages of Experiment 2B. As can be seen in the left panel, Groups RAQ and CMA achieved mean levels around $95 \%$ CRs by the end of Stage 1. As can be seen in the center panel of Figure 5, extinction was complete by the last day of Stage 2. On that day, the highest mean level of responding in any trial block was only $10 \%$ CRs. Any apparent differences between the two groups in either stage were not statistically significant [largest $F(1,19)=2.14$, $p>.10]$.

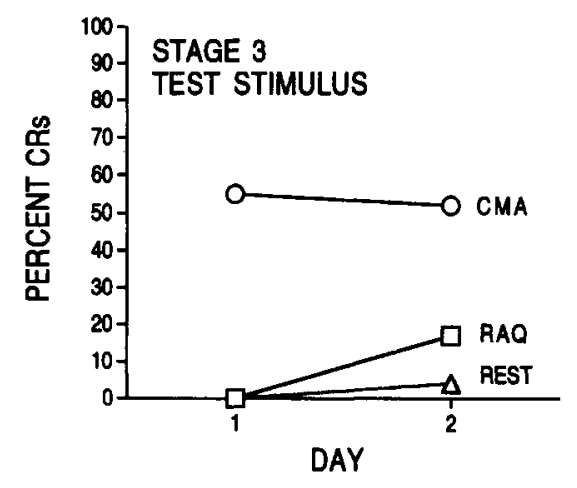

Figure 4. Percentage CRs on tests of the alternative stimulus in Groups RAQ, CMA, and REST as a function of days in Stage 3 of Experiment 2A. 

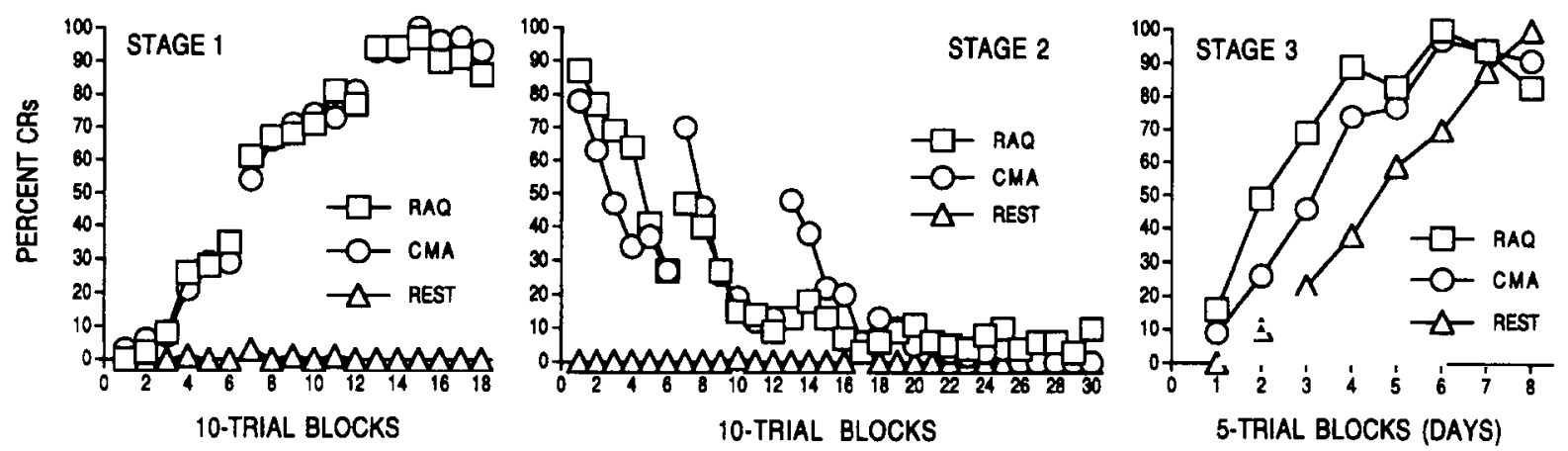

Figure 5. Percentage CRs of Groups RAQ, CMA, and REST in Experiment 2B. For Stages 1 and 2, the data are plotted in 10trial blocks, 6 for each day of training. For Stage 3, the data are plotted in 5-trial blocks, 1 for each day of training.

Figure 5's right panel shows CR acquisition in Stage 3 plotted in 5-trial blocks. Again, Group RAQ's rate of reacquisition appeared faster than cross-modal acquisition in Group CMA. In turn, both of these groups showed a faster rise in responding than Group REST. Statistical comparisons confirmed that Group RAQ ( $M=73 \%$ CRs) showed a higher level of responding than Group REST $(M=48 \%$ CRs $)[F(1,19)=5.51, p<.05]$. Group CMA's results were statistically ambiguous. Group CMA's overall mean level ( $M=64 \% \mathrm{CRs}$ ) failed to differ significantly from that of either Group RAQ or Group REST.

\section{Discussion}

Despite changes in trial spacing after either Stage 1 (Experiment 2A) or Stage 2 (Experiment 2B), the relative performances of the groups in Stage 3 were the same as in Experiment 1 . Reacquisition tended to be slightly faster than cross-modal acquisition, and they both were faster than a rest control. Moreover, in Group CMA in Experiment $2 \mathrm{~A}$, the CR to the original CS showed substantial recovery on nonreinforced test trials during Stage 3.

The pattern of savings was constant across the experiments, thus contradicting predictions that changes in trial spacing would alter the relative retrieval of excitation and inhibition. The fact that the animals' rates of Stage 3 acquisition remained unaffected by contextual change cannot be attributed to a general insensitivity to the manipulation, as was evident in their relative rates of CR acquisition under the different trial spacings. Previously, it has been well documented that reducing the number of trials per session facilitates $C R$ acquisition in the rabbit NM preparation (e.g., Kehoe \& Macrae, 1994). In the present research, initial $C R$ acquisition under spaced training (Experiment 2A) was complete within 45 trials, whereas acquisition to the same level under massed trials required more than 100 trials (Experiments 1 and 2B).

\section{GENERAL DISCUSSION}

The results of these experiments provide an important joint demonstration of two known forms of savings after extinction in classical conditioning of the rabbit NM re- sponse: rapid reacquisition and cross-modal acquisition. In all three experiments, both reacquisition and crossmodal acquisition were more rapid than naive acquisition. Reacquisition was consistently faster than cross-modal acquisition, even though this difference achieved significance in only one out of the three experiments. Moreover, two of the experiments found evidence for a third form of savings: concurrent recovery of the $\mathrm{CR}$ to the original $C S$ when cross-modal acquisition was conducted using a novel CS.

Despite attempts to influence the retrieval of excitatory and inhibitory associations by manipulating the number and spacing of the trials, the pattern of savings was constant across the experiments. The rationale behind changing the number and spacing of trials was to alter contextual factors such that the context of Stage 3 acquisition would be more likely to retrieve inhibitory associations. However, no such contextual effects were seen: Retrieval of excitatory associations appeared to predominate in Stage 3, regardless of the manipulations. An alternative basis for prediction was simply the number of trials used in each stage - that is, the relative number of training and extinction trials presented prior to Stage 3 could be expected to determine the relative strengths of inhibition and excitation influencing acquisition in Stage 3. Contrary to this prediction, in Experiment 2A (spacedmassed-massed), excitatory strength prevailed in Stage 3 regardless of the fact that there had been far fewer initial acquisition trials relative to extinction trials $(1: 12)$.

The pattern of results suggests that, in the rabbit NM preparation, excitatory connections are relatively stable and inhibitory connections are relatively labile following extinction (cf. Pavlov, 1927, p. 66). Further evidence that is consistent with this explanation is that reacquisition tended to be faster than cross-modal acquisition. Conceivably, reacquisition was very rapid because inhibition was easily overthrown, allowing the previously acquired excitatory strength to prevail. In contrast, crossmodal acquisition would be free of the inhibition accruing to the original CS and thus would easily be able to capitalize on preexisting excitatory strength, but it would be slower than reacquisition because of the need to establish 
new associations between the new CS and the US (cf. Kehoe, 1988). Concurrent recovery of the CR to the original CS was very strong, which also indicates that inhibition was easily disrupted, whereas excitation remained stable.

The conclusion that inhibition is relatively labile is not to imply that inhibition is ephemeral in the rabbit NM preparation in general. In fact, inhibitory conditioning has been well demonstrated (e.g., Marchant, Mis, \& Moore, 1972; Marchant \& Moore, 1974; Napier et al., 1992). The lability in inhibition apparent in the present results might have resulted from the use of the 250 -msec CS-US interval, which is optimal for excitatory conditioning of the NM response and less than optimal for inhibitory conditioning. Longer CS-US intervals appear to be less conducive to excitatory conditioning and more conducive to inhibitory conditioning (Hartman \& Grant, 1962; Kehoe \& Graham, 1988; Kehoe \& Schreurs, 1986; Mahoney, Kwaterski, \& Moore, 1975). The future use of longer CS-US intervals might make it possible to replicate Ricker and Bouton's (1996) finding that contextual manipulations can facilitate the retrieval of inhibition during reacquisition and cross-modal acquisition.

The hypothesis that was implicitly adopted in describing the present experiments was that, during extinction, inhibition is superimposed on the excitatory connections underlying the CR; thus, the excitatory connection is not lost, but it is merely masked by the countervailing inhibition (Bouton \& Nelson, 1998; Klopf, 1988; Pavlov, 1927, p. 60). As has been implied throughout the present paper, this "inhibition" hypothesis explains savings after extinction as a shift in the balance of excitation versus inhibition. The shift in the balance between the two kinds of associative strength is often assumed to be precipitated by contextual factors (e.g., Bouton \& Bolles, 1985).

An alternative hypothesis is that, during extinction, excitatory strength is diminished, but a central portion of that excitatory strength remains protected from complete decrement once the CR has disappeared (Kehoe, 1988; Klopf, 1988). Kehoe's (1988) connectionist model, which

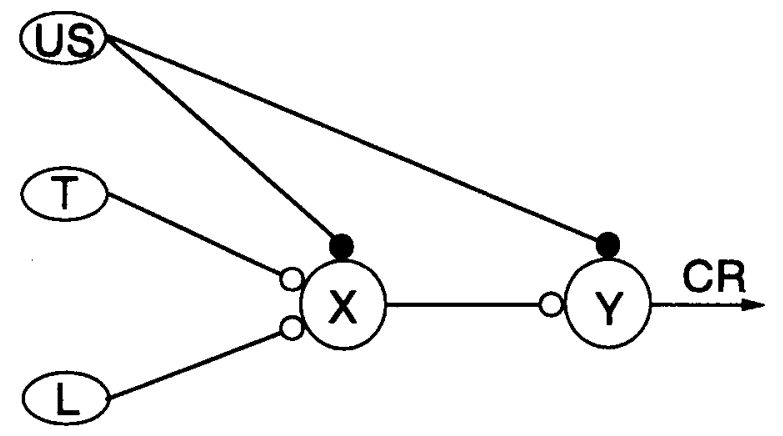

Figure 6. Kehoe's (1988) connectionist model for acquisition, extinction, and savings in classical conditioning. It contains three sensory inputs (US, T, L) and two adaptive units $(X, Y)$. There are plastic excitatory connections between the CS inputs ( $T$ and $L$ ) and the $X$ unit and between the $X$ and $Y$ units. is shown in Figure 6, conceives of CR acquisition as the establishment of two serial associative links. One link is made between the CS input and a "hidden" unit, designated the " $\mathrm{X}$ unit." A second link is made between the $\mathrm{X}$ unit and a response unit, designated the "Y unit," which gives rise to the CR and UR. During CR acquisition to, say, a tone $\mathrm{CS}(\mathrm{T})$, the $\mathrm{T}-\mathrm{X}$ connection is strengthened through presentations of the CS prior to activation of the $X$ unit by the US. The development of an $\mathrm{X}-\mathrm{Y}$ connection depends on the prior establishment of the $T-X$ connection. $\mathrm{CRs}$ to the tone would begin to appear once the $\mathrm{T}-\mathrm{X}$ and $\mathrm{X}-\mathrm{Y}$ connections were both strong enough. In extinction, as the $T-X$ connection becomes too weak to activate the $\mathrm{X}$ unit, the $\mathrm{X}-\mathrm{Y}$ connection would no longer be subject to extinction, and, consequently, the remaining excitatory associations would remain protected from extinction.

Although these two hypotheses agree on the fundamental point that, during extinction, excitatory associations are preserved (e.g., Falls, 1998; McAllister \& McAllister, 1994), they differ in complementary ways with regard to their ability to explain three aspects of the present results. First, the existence of cross-modal transfer is only partly explained by the inhibition hypothesis, which predicts that, in Stage 3 training with a new CS, a change from the context of extinction would create disinhibition, and the resumption of CS-US pairings would cause the retrieval of excitatory associations. However, this hypothesis cannot explain how it is that a new CS can gain access to existing excitatory strength. In contrast, the connectionist model makes the explicit prediction that, once the new CS had established a connection with the $\mathrm{X}$ unit, the residual excitatory $\mathrm{X}-\mathrm{Y}$ connection would quickly be reactivated.

Second, the inhibitory hypothesis does not make any specific predictions regarding the relative rates of reacquisition and cross-modal acquisition after extinction. It simply explains both phenomena as resulting from the dissipation of inhibition that occurs because of contextual change. In contrast, the connectionist model makes a specific prediction that reacquisition should be faster than cross-modal acquisition. In cross-modal acquisition, the $\mathrm{L}-\mathrm{X}$ connection is novel, whereas, in reacquisition, the $\mathrm{T}-\mathrm{X}$ connection has some remaining associative strength and is therefore able to reactivate the $\mathrm{X}-\mathrm{Y}$ connection more rapidly than would the $\mathrm{L}-\mathrm{X}$ connection.

Finally, the inhibition hypothesis is uniquely able to explain concurrent recovery. It would predict that the pairing of a new CS with the US in Stage 3 training would restore US-related aspects of the context of acquisition, thus retrieving excitatory associations. On test trials with the original CS, these excitatory associations would be expressed. Conversely, the connectionist model has no capacity to explain concurrent recovery because there is no way the original CS could regain associative strength without being paired with the US.

One way of capitalizing on the strengths of both of the above hypotheses is to include separate excitatory and inhibitory pathways in a layered network model, as shown 


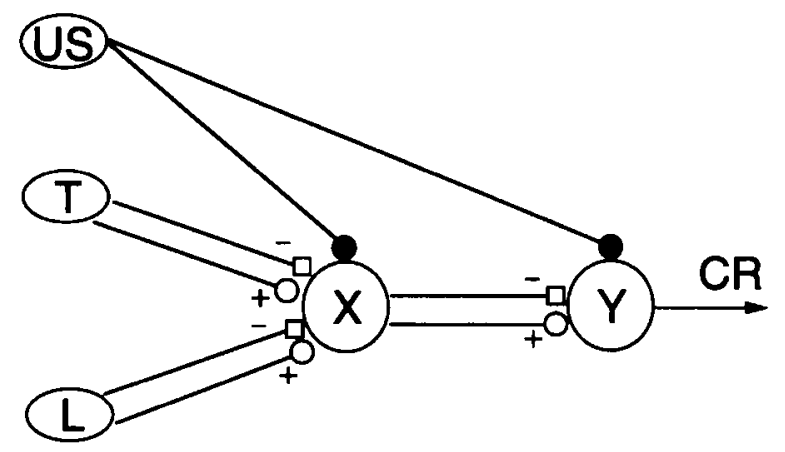

Figure 7. A network for acquisition, extinction, and savings in classical conditioning. It contains three sensory inputs (US, T, L) and two adaptive units $(X, Y)$. There are parallel excitatory and inhibitory plastic connections between the CS inputs ( $T$ and $L$ ) and the $X$ unit and between the $X$ and $Y$ units.

in Figure 7. Like Kehoe's (1988) model, this network receives inputs from a tone CS (T) and a light CS (L) that project to a hidden unit $(\mathrm{X})$ and then to a response unit $(\mathrm{Y})$. The $\mathrm{T}-\mathrm{X}, \mathrm{L}-\mathrm{X}$, and $\mathrm{X}-\mathrm{Y}$ connections consist of parallel excitatory and inhibitory connections, both of which are plastic. It should be noted that this kind of model represents a simplified framework for what, in neural reality, is a complex network of excitatory and inhibitory connections, which actually have multiple parallel connections and multiple sites of plasticity.

According to this "hybrid" model, initial acquisition would entail increases in only the excitatory connections, but, during extinction, both types of connection would change. The excitatory connections would decline, and the inhibitory connections would grow in a negative direction (Klopf, 1988). Output of the X unit and/or Y unit would cease when the algebraic sum of their input connections reaches zero. This balancing of excitation and inhibition would preserve some excitatory strength at the $\mathrm{T}-\mathrm{X}$ and $\mathrm{X}-\mathrm{Y}$ connections, no matter how prolonged the extinction training. During reacquisition training, responding could reappear rapidly on the basis of the residual excitatory strength in both the $\mathrm{T}-\mathrm{X}$ and the $\mathrm{X}-\mathrm{Y}$ connection. Furthermore, a novel CS (L) would still be able to capitalize on the $\mathrm{X}-\mathrm{Y}$ excitatory connection and yield cross-modal transfer. Finally, this model explains concurrent recovery. This prediction depends on the relative stability of the excitatory and inhibitory connections following extinction. If inhibitory connections are relatively labile and context dependent, the residual excitatory connection in an extinguished pathway (e.g., T-X) would regain access to the $X-Y$ connection during crossmodal training with a new CS (e.g., L). This access would become evident as the $\mathrm{X}-\mathrm{Y}$ connection regains its former strength. When the original CS (T) is tested, it would increasingly evoke CRs without having received any further pairings with the US.

From a neural perspective, findings obtained in lesion studies using the rabbit NM preparation provide support for the development of a hybrid model that incorporates both excitatory and inhibitory elements. Specifically, lesions to either the cerebellar cortex (Perrett \& Mauk, 1995 ) or the hippocampus (Akase, Alkon, \& Disterhoft, 1989; Berger \& Orr, 1983; Moyer, Deyo, \& Disterhoft, 1990; Schmaltz \& Theios, 1972) impede extinction of the CR. Following these lesions, rabbits continue to perform the CR even after large numbers of CS-alone trials. This suggests that those sites are involved in inhibitory pathways that are separate to some degree from the excitatory pathways, as envisaged in the model. Moreover, the continuation of the CR in the apparent absence of inhibition indicates that the excitatory connections undergo relatively little diminishment during extinction, which is consistent with our earlier conclusions regarding the persistence of responding in our present experiments. The principle that could be incorporated in the hybrid model is that, while inhibitory connections may be relatively labile, they appear to play a greater role in the extinction of CRs than the diminishment of excitatory connections.

\section{REFERENCES}

AKaSe, E., Alkon, D. L., \& Disterhoft, J. F. (1989). Hippocampal lesions impair memory of short delay conditioned eye blink in rabbits. Behavioral Neuroscience, 103, 935-943.

BERGER, T. W., \& ORR, W. B. (1983). Hippocampectomy selectively disrupts discrimination reversal conditioning of the rabbit nictitating membrane response. Behavioural Brain Research, 8, 49-68.

Bouton, M. E. (1986). Slow reacquisition following extinction of conditioned suppression. Learning \& Motivation, 17, 1-15.

Bouton, M. E. (1993). Context, time, and memory retrieval in the interference paradigms of Pavlovian conditioning. Psychological Bulletin, 114, 80-99.

Bouton, M.E., \& BolLes, R. C. (1985). Contexts, event-memories and extinction. In P. D. Balsam \& A. Tomie (Eds.), Context and learning (pp. 133-166). Hillsdale, NJ: Erlbaum.

Bouton, M. E., \& Nelson, J. B. (1998). The role of context in classical conditioning: Some implications for cognitive behavior therapy. In W. O'Donohue (Ed.), Learning and behavior therapy (pp. 59-84). Boston: Allyn \& Bacon.

FALls, W. A. (1998). Extinction: A review of theory and evidence suggesting that memories are not erased with nonreinforcement. In W. O'Donohue (Ed.), Learning and behavior therapy (pp. 205-229). Boston: Allyn \& Bacon.

Gormezano, I. (1966). Classical conditioning. In J. B. Sidowski (Ed.), Experimental methods and instrumentation in psychology (pp. 385420). New York: McGraw-Hill.

Gormezano, I., \& GibBs, C. M. (1988). Transduction of the rabbit's nictitating membrane response. Behavior Research Methods, Instruments, \& Computers, 20, 18-21.

Gormezano, I., Kehoe, E. J., \& Marshall, B. S. (1983). Twenty years of classical conditioning research with the rabbit. In J. M. Sprague \& A. N. Epstein (Eds.), Progress in psychobiology and physiological psychology (Vol. 10, pp. 197-275). New York: Academic Press.

Hart, J. A., Bourne, M. J., \& Schachtman, T. R. (1995). Slow reacquisition of a conditioned taste aversion. Animal Learning \& Behavior, 23, 297-303.

Hartman, T. F., \& Grant, D. A. (1962). Differential eyelid conditioning as a function of the CS-US interval. Journal of Experimental Psychology, 64, 131-136.

KEHOE, E. J. (1988). A layered network model of associative learning Learning-to-learn and configuration. Psychological Review, 95, 411433.

KeHOE, E. J., Feyer, A.-M., \& Moses, J. L. (1981). Second-order conditioning of the rabbit's nictitating membrane response as a function of the CS2-CS1 and CS1-US intervals. Animal Learning \& Behavior, 9, 304-315 
Kehoe, E. J., \& Graham, P. (1988). Summation and configuration in negative patterning of the rabbit's conditioned nictitating membrane response. Journal of Experimental Psychology: Animal Behavior Processes, 14, 320-333.

KeHOE, E. J., Horne, A. J., \& MACRAE, M. (1995). Learning to learn: Real-time features and a connectionist model. Adaptive Behavior, 3 , 235-271.

KEHOE, E. J., \& MACRAE, M. (1994). Classical conditioning of the rabbit nictitating membrane can be fast or slow: Implications for Lennartz and Weinberger's (1992) two-factor theory. Psychobiology, 22, 1-4.

KeHOE, E. J., \& MACRAE, M. (1997). Extinction, reacquisition, and cross-modal transfer in conditioning of the rabbit's nictitating membrane response. Society for Neuroscience Abstracts, 23, 782.

KEHOE, E. J., MORROW, L. D., \& HolT, P. E. (1984). General transfer across sensory modalities survives reductions in the original conditioned reflex in the rabbit. Animal Learning \& Behavior, 12, 129-136.

Kehoe, E. J., \& Schreurs, B. G. (1986). Compound-component differentiation as a function of CS-US interval and CS duration in the rabbit's conditioned nictitating membrane response. Animal Learning \& Behavior, 14, 144-154.

KLOPF, A. H. (1988). A neuronal model of classical conditioning. Psychobiology, 16, 85-125.

Mahoney, W. J., Kwaterski, S. E., \& Moore, J. W. (1975). Conditioned inhibition of the rabbit nictitating membrane response as a function of CS-UCS interval. Bulletin of the Psychonomic Society, $5,177-179$

Marchant, H. G., Mis, F. W., \& Moore, J. W. (1972). Conditioned inhibition of the rabbit's nictitating membrane response. Journal of Experimental Psychology, 95, 408-411.

Marchant, H. G., \& MoORE, J. W. (1974). Below-zero conditioned inhibition of the rabbit's nictitating membrane response. Journal of Experimental Psychology, 102, 350-352.

Marshall-Goodell, B., Schreurs, B. G., \& Gormezano, I. (1982). Ruler vs. the Apple II/FIRST system analysis of analog signals in classical conditioning. Behavior Research Methods \& Instrumentation, 14, 519-525.

MCALlister, D. E., \& MCALLISTER, W. R. (1994). Extinction and reconditioning of classically conditioned fear before and after instrumental learning: Effects of depth of fear extinction. Learning \& $\mathrm{Mo}$ tivation, 25, 339-367.

MOYER, J. R., Deyo, R. A., \& DisterhoFT, J. F. (1990). Hippocampectomy disrupts trace eye-blink conditioning in rabbits. Behavioral Neuroscience, 104, 243-252.

Napier, R. M., Macrae, M., \& KehoE, E. J. (1992). Rapid reacquisition in conditioning of the rabbit's nictitating membrane response Journal of Experimental Psychology: Animal Behavior Processes, 18, 182-192.

Pavlov, I. P. (1927). Conditioned reflexes: An investigation of the physiological activity of the cerebral cortex (G. V. Anrep, Trans.). London: Oxford University Press.

Perrett, S. P., \& MauK, M. D. (1995). Extinction of conditioned eyelid responses requires the anterior lobe of the cerebellar cortex. Journal of Neuroscience, 15, 2074-2080.

Revusky, S., \& CoOmbes, S. (1979). Reacquisition of learned taste aversions. Animal Learning \& Behavior, 7, 377-382.

Ricker, S. T., \& Bouton, M. E. (1996). Reacquisition following extinction in appetitive conditioning. Animal Learning \& Behavior, 24 , 423-436.

SCANDRETT, J., \& GoRmEZANO, I. (1980). Microprocessor control and A/D data acquisition in classical conditioning. Behavior Research Methods \& Instrumentation, 12, 120-125.

Schmaltz, L. W., \& Theios, J. (1972). Acquisition and extinction of a classically conditioned response in hippocampectomized rabbits (Oryctolagus cuniculus). Journal of Comparative \& Physiological Psychology, 79, 328-333.

(Manuscript received October 15, 1997 revision accepted for publication June 23, 1998.) 